\title{
A Cable-based Series Elastic Actuator with Conduit Sensor for Wearable Exoskeletons
}

\author{
Laura H. Blumenschein, Student Member, IEEE, Craig G. McDonald, Student Member, IEEE, and \\ Marcia K. O’Malley, Senior Member, IEEE
}

\begin{abstract}
There is currently a scarcity of wearable robotic devices that can practically provide physical assistance in a range of real world activities. Soft wearable exoskeletons, or exosuits, have the potential to be more portable and less restrictive than their rigid counterparts. In this paper, we present the design of an actuation system that has been optimized for use in a soft exosuit for the human arm. The selected design comprises a DC motor and gearbox, a flexible cable conduit transmission, and a custom series elastic force sensor. Placed in series with the transmission conduit, the custom compliant force sensor consists of a translational steel compression spring with a pair of Hall effect sensors for measuring deflection. The custom sensor is validated as an accurate means of measuring cable tension, and it is shown that it can be used in feedback to control the cable tension with high bandwidth. The dynamic effect of the cable-conduit transmission on the force felt at the user interface is characterized by backdriving the system as it renders a range of virtual impedances to the user. We conclude with recommendations for the integration of such an actuation system into a full wearable exosuit.
\end{abstract}

\section{INTRODUCTION}

In recent decades we have witnessed the transition of wearable robotic devices from concept to reality. Rigid robotic exoskeletons have a number of practical limitations due to their physical intrusiveness, difficulties in transport, and kinematic constraints, limiting their use to highly controlled laboratory or clinical settings [1]. There is currently a scarcity of wearable robotic devices that can practically provide physical assistance in a range of real world activities. Soft wearable exoskeletons, or exosuits, have been introduced to address these issues. These exosuits forgo large, bulky support structures, instead relying on the human skeletal system to provide joints as well as to support compressive loads resulting from actuation. Though this design decision can limit their overall output strength and speed, the soft exoskeleton concept has been successfully realized as robotic gloves [2]-[5], soft orthoses [6], lower limb devices for metabolic reduction [7], [8] and rehabilitation [9].

Successful actuators for soft exoskeletons have been application specific and fall largely into two groups: pneumatic muscles and cable-driven remote actuation. Low-force

This work was funded in part by NASA grant NNX14AK51G, NSF DGE1250104, and NSF GRFP-0940902

Laura Blumenschein is with the Department of Mechanical Engineering, Stanford University, Stanford, CA, 94035 USA and formerly with the Mechatronics and Haptic Interfaces Laboratory at Rice. Craig G. McDonald and Marcia K. O'Malley are with the Mechatronics and Haptic Interfaces Laboratory, Department of Mechanical Engineering, Rice University, Houston, TX 77005. (e-mail: lblumens@ @stanford.edu)

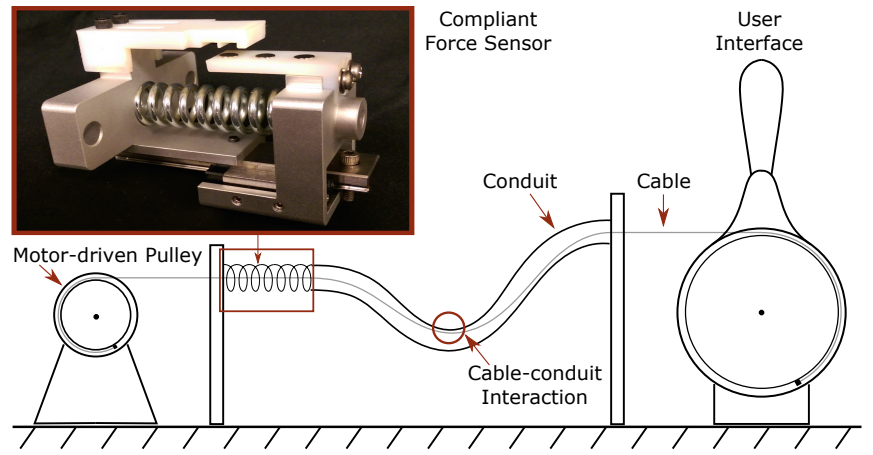

Fig. 1. Actuator concept showing motor-driven pulley, cable-conduit transmission with series elasticity and a stand-in user interface. An example of cable-conduit interaction can be seen at the bends, which is responsible for the transfer of cable tension into conduit compression. Image callout: Compliant force sensor prototype with magnets visible, embedded in 3Dprinted white plastic at top.

coordinated movements in gloves have commonly been accomplished by pressurized McKibben artificial muscles or soft pressurized elastomeric cylinders [5]. Higher force applications, however, have almost exclusively been approached with cable-driven devices. A device designed by Asbeck et al., which actuates the hip and ankle during walking, initially used pneumatic actuation but was later redesigned to utilize Bowden cables, a type of felxible cable-conduit transmission [10]. Outside of lower limb and hand, few actuators have been designed and implemented that focus on soft exoskeletons for the arm. Kong et al. have proposed multiple designs for cable driven actuators, but with a focus on tethered, over mobile, operation [11].

This class of soft exoskeleton actuators use compliance in non-actuated directions to maintain joint alignment and be less intrusive to the user. Additionally, compliance in series with the actuation path can improve the safety and quality of physical interactions. First proposed by Pratt and Williamson [12], series elastic actuation (SEA) is a design strategy and method of force control that places intentional compliance in the actuator load path to provide additional safety to user interaction, improved shock tolerance, improved contact stability, and reduced reflected inertia. For soft exosuit applications, series elastic actuators provide a stable means of force and impedance control given the intrinsic compliance of Bowden cable transmissions and textiles-based robotuser interface points. Series elastic actuation has previously been combined with cable-conduit actuators in rigid robotic devices [13], [14] and recently some designs by Cappello 
et al. for a non-linear SEA for an elbow exosuit have been proposed but not fully validated [15].

In this paper, we present the design of a series elastic actuator for use in a soft assistive exosuit for shoulder and elbow. Unlike previous Bowden cable SEAs, the design will be optimized for upper arm rehabilitation and fit within the design motivations for soft exosuits.

\section{DESIGN FACTORS}

In this section we will explore how the need for exosuits to be portable and wearable during activities of daily living is translated into specific actuator design considerations. First and foremost, any restrictions on natural human range of motion while wearing the device should be kept to a minimum. Seeing as even casual clothing can in some cases restrict motion, we must expect an exoskeleton to restrict motion to some extent. A good goal is for the device to achieve the range of motion (ROM) required to complete most activities of daily living, which is a relatively small subset of the joint space achievable by healthy persons.

Second, any additional mechanical impedance introduced while wearing the device should also be kept to a minimum. Mechanical impedance is a measure of how much a structure resists motion. When unpowered, the passive impedance of the device is a combination of the impedance due to the placement and weight of the components contacting the wearer and the impedance from backdriving the actuators. Once a device is powered and the actuators are producing forces to either assist or resist the wearer, the device is then actively modulating its own impedance from the human's perspective. The modulated impedance seen by the user is often called the virtual impedance. The lowest virtual impedance that a device can achieve, which is also referred to as the device transparency, is a function of the passive impedance and the controller performance, and will always have a lower bound greater than zero [16].

Several members of the robotics community have arrived at the same solution for an actuator design that minimizes these negative properties. The common features of this design are a DC motor and gearbox, a flexible cable-conduit transmission, and some physical interface that transfers cable loads to the wearer's limbs. The DC motor is ubiquitous in the field of robotics, its dynamics are well understood, and it can have extremely high power to weight/volume ratio. Despite their high power density, the weight of a motor can still be significantly detrimental when attached to a human limb. On lower limb experiments, even small amounts of weight placed on the limb increase metabolic costs, and the effect increases significantly as weight is placed more distally [17]. This makes direct drive motors, which must be placed directly at the joint, an unsuitable choice. The benefit of the cable-conduit transmission is to relocate the motors away from the limb onto a more central and stationary part of the body where they also will no longer interfere with the workspace.

When examining the design of exoskeletons that utilize this cable-conduit actuation strategy, we can see that designs are tailored to the part of the body that is to be actuated. The factors of interest when tailoring the actuation system are work space complexity, force range, and control accuracy. Devices for the lower limb benefit from the workspace being notably limited, as gait is repetitive and fairly uncomplicated in a kinematic sense. Therefore, we see successful implementation of designs such as the LOPES, where bulky but light structures can be added that allow for robust force control [11], [13]. Soft exosuits developed for the lower limb are highly effective and do manage to keep an extremely low profile that interferes minimally with activity, but they are not able to provide the same level of forces as rigid exoskeletons [10]. Similarly, the requirements on the accuracy and consistency of a cable force controller for such devices can be relatively lax compared with the requirements for upper limb devices. For example, an invariant trapezoidal force profile triggered once per gait cycle has been found sufficient to reduce metabolic costs during walking [18].

On the opposite end of the design spectrum lie devices for the hand. In this regime, minimizing bulkiness and self collision are important with higher degree-of-freedom movements in a smaller space. Additionally, force control in gloves offers the opposite challenge from lower limb applications, needing to be extremely accurate to provide useful assistance, but at significantly lower force levels. One example of a cable driven hand exoskeleton uses the principle of series elastic actuation to measure and control forces applied to the individual finger joints in addition to a DC motor with cable-conduit transmission design [14]. Similar series elastic actuation is also seen in some lower limb devices, such as LOPES et al. [11], [13].

The set of design considerations for an arm exosuit lies between these two extremes. Accurate force control is required over a complex joint space like seen in gloves but with force levels closer to those used in lower limb devices. To achieve higher force control accuracy, we propose using actuator hardware similar to soft lower limb exosuits but utilizing the force control capabilities of series elastic actuation. We have integrated an elastic element in series with the cable-conduit actuator, located in series with the cable. It is placed in the conduit and mounted on the same side of the transmission as the motor. This actuator is similar to the rigid LOPES SEA mentioned above for lower limb but is focused in the choice of compliant element, sensors, and other elements to a soft arm exosuit.

\section{Cable Actuation System with Series ELASTICITY}

\section{A. Series Elastic Actuator Requirements}

The requirements for the series elastic actuator for a soft arm exosuit fall into two categories: the qualitative driving design considerations for soft exosuit actuators as discussed in Section II and the specific human capabilities that must be matched. While it is not our aim to exceed human capabilities in force and torque generation, for the purposes of rehabilitative or assistive devices, providing sufficient joint torque and speed to match human abilities during activities 


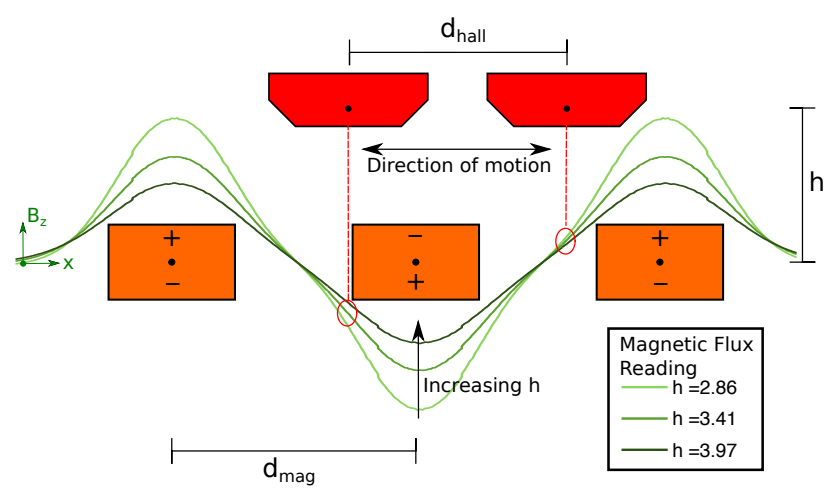

Fig. 2. Hall effect based translational position sensor using a novel three magnet, two sensor design. Relative positioning of the magnets (orange) and Hall effect sensors (red) are shown. Magnets are equally spaced by a distance $d_{m a g}$, and Hall effect sensors separated by $d_{h a l l}$. The sensors translate relative to the magnets at a constant height of $h$ above the magnets. Example magnetic flux distributions are shown in green. Peak flux magnitude coincides with the center of each magnet and the sign of the peak depends on the direction of the magnetic dipole. The peak value drops with increasing $h$ but linearity between the peaks increases. The two sensors read from the same distribution but read at locations offset by the sensor separation $d_{\text {hall }}$.

of daily living is necessary. Torques necessary for normal elbow and shoulder movements during ADLs are below $4 \mathrm{~N}$ $\mathrm{m}$ and $10 \mathrm{~N}-\mathrm{m}$ respectively and maximum joint speeds for both joints are $170 \mathrm{deg} / \mathrm{s}$ [19].

Besides the motor, the compliant element within the series elastic actuator also faces certain requirements. The compliant element has three design specifications: force range, stiffness, and footprint. In addition to the torque requirements above, we can draw upon biological inspiration for stiffness requirements. Designing with these parameters in mind ensures that the system will be able to measure the full range of forces needed for the application and that it will at the same time have a stiffness appropriately matched to that of the human elbow and shoulder joint. For the stiffness of the compliant element we choose a stiffness on the order of the maximum stiffness of the human elbow and shoulder, about $15 \mathrm{~N}-\mathrm{m} / \mathrm{rad}$ [20]. These biologically inspired requirements are sufficient to define the SEA.

\section{B. Actuator Components}

To meet the requirements for soft exoskeleton design targeting the upper limb, we propose the following series elastic actuator design: a cable-conduit actuator with in-line compliance. We selected a 200 W, 48 V EC-4 Pole Maxon Motor with a 246: 1 planetary gearbox and 500 counts per revolution optical encoder. The motor combination can provide a continuous torque of $8 \mathrm{~N}-\mathrm{m}$, with intermittent torques up to $12 \mathrm{~N}-\mathrm{m}$, and a continuous speed of $195 \mathrm{deg} / \mathrm{s}$. A flexible cable-conduit transmission, or Bowden cable, relocates the motor away from the load. The transmission consists of a Vectran ${ }^{\mathrm{TM}}$ cable coupled with a coiled steel conduit with Teflon ${ }^{\mathrm{TM}}$ lining, providing low friction and resistance to cable creep. To keep the actuator package small and limit the need for additional gearing at the user interface, the lever arm for the motor to cable interface was kept small with a gearbox pulley of $36 \mathrm{~mm}$.

A translational compliant force sensor provides the force measurement and linear compliance of the series elastic actuator. Fig. 1 depicts the placement of the compliant sensor in series with the conduit. This sensor, pictured in Fig. 1 inset, is placed collinear with the Bowden conduit, a novel placement of compliant force sensors in cable-driven actuators. This placement therefore does not interfere with a natural range of motion or add significant impedance to the user and meets the requirements of soft exosuit design. Translating the biologically inspired force and stiffness requirements to the translational domain through the motor pulley gives a desired maximum force of $277.8 \mathrm{~N}$ and a desired stiffness of $115.7 \mathrm{~N} / \mathrm{cm}$. A steel compression spring with a reported spring rate of $169.7 \mathrm{~N} / \mathrm{cm}$ and maximum load of $365.96 \mathrm{~N}$ was chosen (McMaster PN: 9657K395), matching well with the biologically inspired requirements while allowing some factor of safety. A stationary translational compression sensor design allows for a direct measurement of the compliant element compression with a simple design, reducing error due to noise [21]. The spring deflection is measured using a custom designed non-contact translational position sensor.

1) Compliant Sensor Design: A novel translational position sensor design was developed and tested using three neodymium magnets, grade N52 with $4.8 \mathrm{~mm}$ diameter and $1.59 \mathrm{~mm}$ height, and two Hall effect sensors, $\pm 100 \mathrm{mT}$ range. The design of the sensor is an extension of the Hall effect based sensor presented by Sergi et al. [22]. To design the custom Hall effect sensor, we simulated the magnetic fields using the 2D freeware magnetic simulator FEMM. The FEMM simulation calculated the z-direction magnetic flux at every point in space for axisymmetric magnetic fields of a single cylindrical magnet. These magnetic flux values were shifted in the $x$ direction to match the proposed magnet position and superimposed to predict the flux readings for the multi-magnet design. A 2D representation of the general translational position sensor design is displayed in Fig. 2. The three design variables, magnet separation $\left(d_{m a g}\right)$ sensor separation $\left(d_{\text {hall }}\right)$ and magnet to sensor height $(h)$ define the magnetic field and the locations in the flux field read by the Hall effect sensors. Since the sensor translates only in one

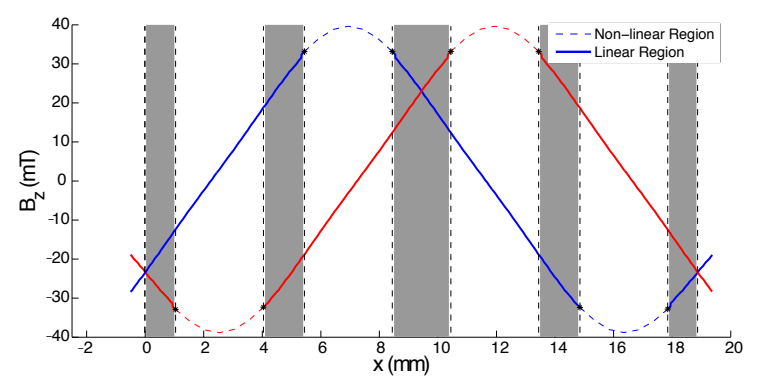

Fig. 3. Simulation of readings from two Hall effect sensors (one in red and one in blue) for $d_{\text {hall }}$ equal to $5 \mathrm{~mm}, d_{\text {mag }}$ equal to $9.4 \mathrm{~mm}$, and $h$ equal to $3.97 \mathrm{~mm}$. Solid lines indicate usable linear regions and vertical divisions mark the shaded regions where both sensors read in linear regions. 

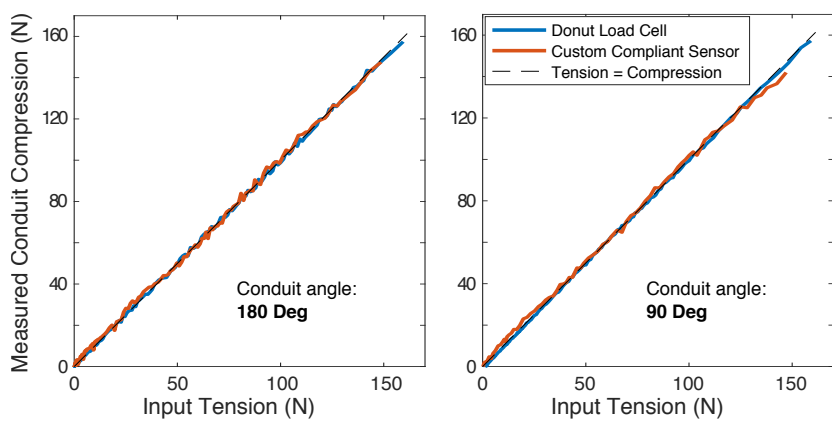

Fig. 4. Evaluation of conduit compression force measurement as a measure of cable tension at two conduit wrap angles: $90 \mathrm{deg}$ and $180 \mathrm{deg}$. Force read by the custom compliant conduit force sensor and a commercially available donut load cell at the same location was plotted against force read by a different commercial force sensor measuring cable tension directly, over the full range of the custom sensor. Maximum error of the sensors was $1.99 \%$ FSO for the custom sensor and $1.09 \%$ FSO for the donut load cell.

degree of freedom above the centerline of the magnets at a constant height, we need only consider the 1D magnetic flux simulation examples shown in green in Fig. 2 .

The design was optimized with the goal of maximizing the full range of the sensor while limiting the sensor error and non-linearity. To read the full spring compression, the sensor needs a range of $21.6 \mathrm{~mm}$. For sensor error, similar sensor designs have used $3-5 \%$ of full state output as an acceptable error limit [22]. To achieve this optimization, the magnetic field and the 1D slice read by a single sensor were designed. Magnet separations, $d_{\text {mag }}$, between $7.5 \mathrm{~mm}$ and $10.5 \mathrm{~mm}$ were tested with sensor read heights, $h$, between $2.58 \mathrm{~mm}$ and $4.25 \mathrm{~mm}$. A value of $d_{m a g}=9.4 \mathrm{~mm}$ maximizes the period length of the magnetic flux while retaining a large viable range of $h$ values. High linearity and high sensitivity both increase the final performance of the sensor within the readable regions. For the range of $h$ values, linearity and sensitivity metrics are summarized in Table I. The error calculated from the simulation is a conservative theoretical maximum, so it is important to choose a value of $h$ that predicts error significantly lower than the desired $3 \%$ of Full Scale Output (FSO). A value for $h$ of 3.97 maximizes the linearity without reaching the lowest sensitivity simulated.

The range was then extended by choosing $d_{\text {hall }}$ to create

TABLE I

SimUlated LINEARITY AND SENSITIVITY FOR TRANSLATIONAL Position SEnsor VARYing Magnet Height

\begin{tabular}{c||c||c||c}
\multicolumn{1}{c||}{$h(\mathbf{m m})$} & $\boldsymbol{R}^{\mathbf{2}}$ & $\begin{array}{c}\text { Max Error } \\
(\boldsymbol{\%} \text { FSO })\end{array}$ & $\begin{array}{c}\text { Sensitivity } \\
(\mathbf{m T} / \mathbf{m m})\end{array}$ \\
\hline 2.58 & 0.9916 & $4.81 \%$ & 20.6 \\
\hline 2.86 & 0.9955 & $3.91 \%$ & 17.6 \\
\hline 3.13 & 0.9977 & $2.92 \%$ & 15.0 \\
\hline 3.41 & 0.9990 & $2.16 \%$ & 12.8 \\
\hline 3.69 & 0.9996 & $1.80 \%$ & 11.1 \\
\hline 3.97 & 0.9999 & $0.90 \%$ & 9.68 \\
\hline 4.25 & 0.9999 & $1.22 \%$ & 8.33
\end{tabular}

a series of linear measurement regions across the full sensor range that can be uniquely identified by the relative readings of the two sensors (Fig. 3). The max value for $d_{\text {hall }}$ was $6.4 \mathrm{~mm}$ as any larger caused regions with only non-linear readings to exist, while the separation could only be as small as $5 \mathrm{~mm}$ due to manufacturability. The minimum value of $5 \mathrm{~mm}$ was chosen in order to have maximal overlap of the linear regions for improved robustness. The magnetic flux readings resulting from optimization of the sensor geometry are simulated in Fig. 3.

This novel position sensor was integrated with the compression spring into a compliant force sensor as seen in Fig. 1. The final design consists of two aluminum mounting blocks fixed to either side of the spring. The two halves of the sensor are attached to a slider bearing so they may translate independently of each other, allowing practically all of the load on the sensor to work toward compressing the spring. Each mounting block attaches to one component of the displacement sensor - either the magnets or the Hall effect sensors - and has an interface to the conduit cable transmission. The cable conveniently runs through the hollow cavity of the compression spring.

2) Compliant Sensor Calibration: We developed a calibration method for the translational position sensor. The linear regions of the two sensor ranges can be combined to form a piecewise linear function covering the full sensor domain. Using the values of both sensors, the correct region of the piecewise function can be uniquely chosen, and the sensor displacement calculated. In the striped areas of Fig. 3, where both sensors lie in a linear region, the resulting displacement calculations are averaged to give a more accurate measurement.

For each sensor, the reading is checked against the opposing sensor reading and the upper and lower linear limits to find the occupied linear region according to the following algorithm:

Input: Values of sensor of interest, CurrVal, and opposing sensor, OppVal

Output: Linear region the sensor of interest is reading within

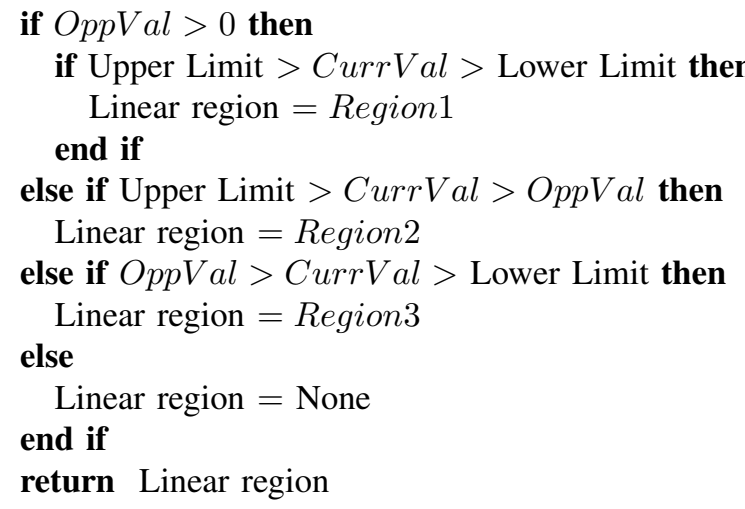

In this way the values for slope, $m_{i}$, and intercept, $b_{i}$, are selected among the three linear regions for each sensor, and a value for $c_{i}$ is set to one or zero indicating if the sensor is reading in a linear or non-linear range. These coefficients are 


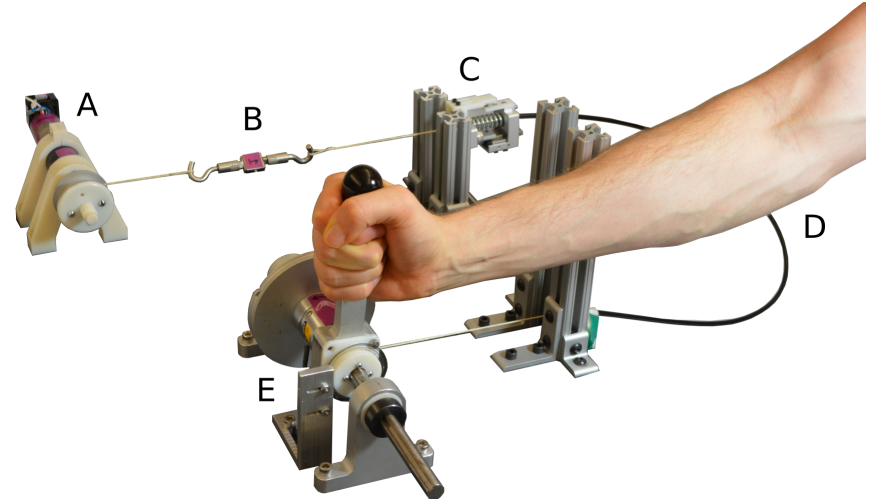

Fig. 5. Experimental test bed for series elastic cable-conduit actuator to drive soft exosuits for the arm. Actuation system includes a DC motor (A), cable-conduit transmission (D), and series elastic sensor (C). Other components that are not part of the actuator architecture but are needed for actuator validation are the non-compliant cable tension sensor (B) and the user interface with position and force sensing (E).

used to calculate the corresponding displacement as follows:

$$
x=\frac{c_{1}\left(m_{1} s_{1}(x)+b_{1}\right)+c_{2}\left(m_{2} s_{2}(x)+b_{2}\right)}{c_{1}+c_{2}}
$$

where $s_{1}(x)$ and $s_{2}(x)$ are the sensor outputs.

3) Compliant Sensor Validation: The placement of the custom compliant force sensor as depicted in Fig. 1 was confirmed as an accurate way to measure cable tension. With the placement in the conduit, the sensor indirectly measures the cable tension through the resulting conduit reaction forces. This method is valid as long as there is some interaction between the cable and conduit, i.e. the transmission angle is not zero. Using a commercial tension sensor (Futek LSB200), the force versus position relationship of the sensor was found. A linear fit between the sensor displacement and measured force was calculated with an $R^{2}$ value of 0.9998 , yielding the compliant sensor stiffness of $177.1 \mathrm{~N} / \mathrm{cm}$. In Fig. 4, the relationship between cable tension and conduit sensor measurement is shown for the custom compliant force sensor with two conduit wrap angles, to show the independence of the force measurement from transmission configuration. A non-compliant commercially available sensor was tested in the same configuration for reference. The compliant conduit force sensor's maximum error of $1.99 \%$ FSO is well within the desired error bounds of previous compliant sensors and only twice the error of the commercially available rigid sensor, 1.09\% FSO.

\section{Design Validation}

We characterize our ability to control cable tension at the custom sensor location ( $\mathrm{C}$ in Fig. 5) using its force measurement in feedback. We then characterize the dynamic effect of the cable-conduit transmission frictional loss on the ability to control the user interaction force while rendering a virtual impedance.
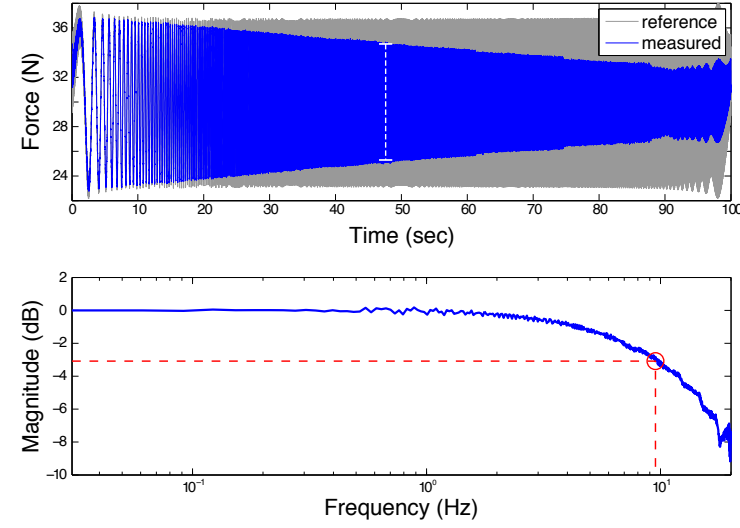

Fig. 6. Closed-loop force tracking performance characterized with a Schroeder multisine input waveform with a frequency range of $0-20 \mathrm{~Hz}$ and the magnitude of the corresponding transfer function plotted below. The force tracking bandwidth is approximately $9.5 \mathrm{~Hz}$, as indicated by the dashed lines.

\section{A. Force Control}

Actuator force output was controlled using a cascaded control scheme, where an outer loop with proportional cable force feedback commands motor angular velocities to an inner loop with proportional and integral (PI) velocity control, in the style of [23], illustrated in Fig. 7. We characterize the actuation system's ability to control cable tension at the motor side of the conduit using the custom compliant sensor as the source of the force feedback signal, without any user-generated disturbances present, i.e., the load handle was locked in place. Using a standard system identification technique, we applied a Schroeder multisine waveform with a mean of $30 \mathrm{~N}$ and amplitude of $8 \mathrm{~N}$ to excite frequencies in the range of $0-20 \mathrm{~Hz}$. Using the command waveform and the measured force at the custom sensor, the magnitude of the transfer function representing closed-loop force control was estimated using the Welch method. The resultant bandwidth, highlighted in Fig. 6, is approximately $9.5 \mathrm{~Hz}$, which is comparable to similar devices [22]. The compliance of the actuator acts to lessen the force bandwidth of the system as compared to a rigid actuator [24].

\section{B. Impedance Control}

Impedance control is the simplest human-robot interaction controller that satisfies our minimum requirements of safe, stable assistance along a desired trajectory; it allows for interaction dynamics to be defined at the human-robot interface instead of merely prescribing a time-varying position or force. Using the following control law:

$$
f_{r}=-k_{v}\left(x_{h}-x_{r}\right)-b_{v} \dot{x}_{h}
$$

we can assist a user in following a trajectory $x_{r}(t)$ by rendering a virtual spring of stiffness $k_{v}$ pulling them back to $x_{r}$ and a virtual damper with damping coefficient $b$ dissipating any oscillatory behavior. An optical encoder rotating with the user interface (see Fig. 5 E) was used to measure the 


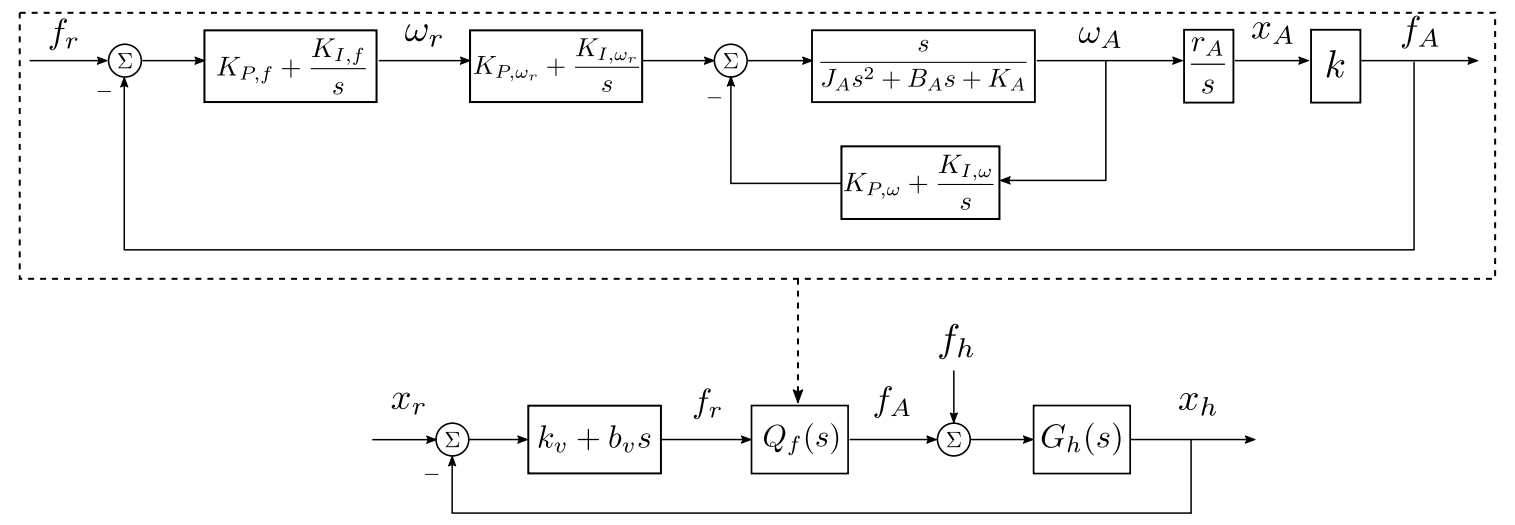

Fig. 7. Cascaded force-velocity controller nested inside an impedance control loop. The closed-loop force controller is shown in the dotted box, with reference force $f_{r}$ and for generated by actuator motion $f_{A}$. Within the force control loop is a velocity controller, which causes the angular velocity of the motor pulley $\omega_{A}$ to track the reference velocity $\omega_{r}$. Parameters $J_{A}, B_{A}$, and $K_{A}$ are the reflected rotational inertia, damping, and stiffness of the actuator at the motor pulley. The proportional and integral gains for the velocity controller were tuned so that the velocity controller acted as a first-order linear system with time constant $T_{\omega}=4.42 \mathrm{~ms}$. The radius of the motor pulley is $r_{A}$, the translation of the cable at the actuator end of the conduit is $x_{A}$, and the actual stiffness of the translational spring is $k$. Closed-loop force control is achieving by using $f_{A}$ in feedback, where the contribution from the user's load motion has been ignored, and proportional and integral gains set to be $K_{P, f}=12$ and $K_{I, f}=0$. In the lower block diagram, the entire closed-loop force controller is represented by the block $Q_{f}(s)$, and the system is drawn coupled with the user dynamics, $G_{h}(s)$, to represent impedance control while the human grasps the user interface. Disturbance forces applied by the human $f_{h}$ combine with $f_{A}$ to produce motion at the user interface $x_{h}$. The cable translation at the user interface $x_{h}$ is compared to a desired reference trajectory $x_{r}$ as input to the impedance controller, which renders a virtual stiffness $k_{v}$ and virtual damping $b_{v}$.

translation at the user end of the cable, $x_{h}$. The cable tension felt by the user was measured using a commercial torque load cell (Futek TFF350) rigidly mounted between the pulley and the handle (Fig. $5 \mathrm{E}$ ), and the cable tension at the actuator side was also measured using a commercial load cell (Futek LSB200, Fig. 5 B).

The actuator was tested with a range of desired stiffness values and zero desired damping. Desired stiffnesses above the actual stiffness of the compliant element were not tested, since it has been shown that rendering virtual stiffnesses above the actual stiffness of an SEA threatens the coupled stability of the user and the robot under our impedance control implementation [23]. In order to isolate the accuracy of impedance rendering, the reference trajectory $x_{r}(t)$ was set to be identically zero. Fig. 8 shows force versus displacement at the user interface as the user is cyclically loading and unloading the cable in a natural way.

The cable force, when measured on the actuator side, very accurately follows the desired high stiffness curve shown in Fig. 8. Using linear regression to determine the average stiffness rendered to the user, it is also accurate for the low value of stiffness shown in Fig. 8, but there is noticeable hysteresis, suggesting viscous damping is present. This is expected, as Losey et al. [25] observed, when an SEA is rendering a virtual stiffness that is near its actual stiffness, the required motion of the actuator is minimal; conversely, when the virtual stiffness is much lower, the actuator must quickly move in a way that reduces actual spring deflection. Therefore, when the virtual environment being rendered requires quick actuator movements, viscous friction in the system that could not be eliminated fully by our control architecture.

When we examine the cable tensions measured at the user interface we also see significant hysteresis, but the shape of the curve no longer suggests just viscous friction. Instead, the frictional forces caused by cable-conduit interaction, which follow the well-known "capstan equation" [26], lead to highly nonlinear behavior [27]. The stiffness rendered to the user is effectively higher as they are loading the cable, and the stiffness feels lower as the cable is being unloaded. As the user reverses direction, static friction accounts for the nearly vertical transitions between the two stiffnesses, which resembles backlash. Using only the actuator-side cable force measurement in feedback, this cable-conduit frictional effect can only be mitigated by predictive compensation, either model-based or learned.

\section{Conclusion}

This work has introduced a novel design for an actuation system to be used in soft wearable robotic exosuits for the upper limbs. After analysis of existing soft exoskeleton designs and cable-driven devices both for lower limb and hand, the common design influences of these solutions were synthesized for the unique problem of exosuits for the upper limb. To best balance work space complexity, force control performance, and force range requirements, series elastic actuation of a flexible cable-conduit system is proposed. The selected actuator design includes a custom compliant force sensor placed in series with the conduit, which has a sensor range, resolution, and compliance optimized for the upper limb application. Force control and impedance control at the sensor were successfully demonstrated, but the effects of cable-conduit friction were noticeable when looking at the impedance rendered to the user.

Mounting the sensor at the motor side of the conduit is advantageous because 1) the loading path from sensor 

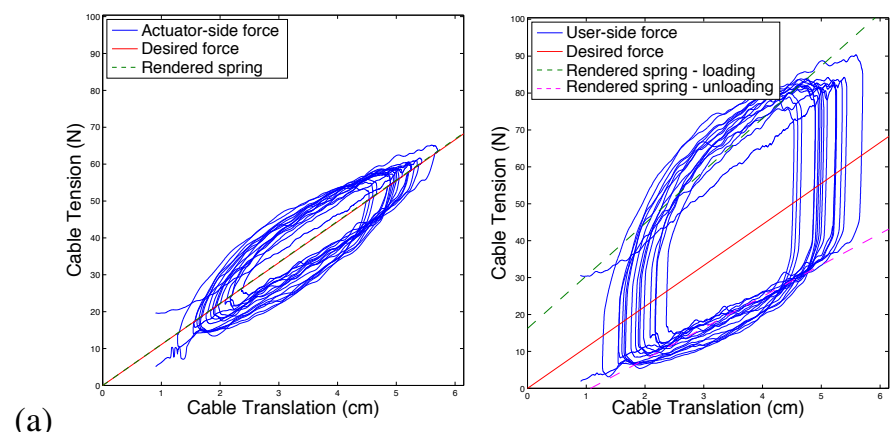

(a)
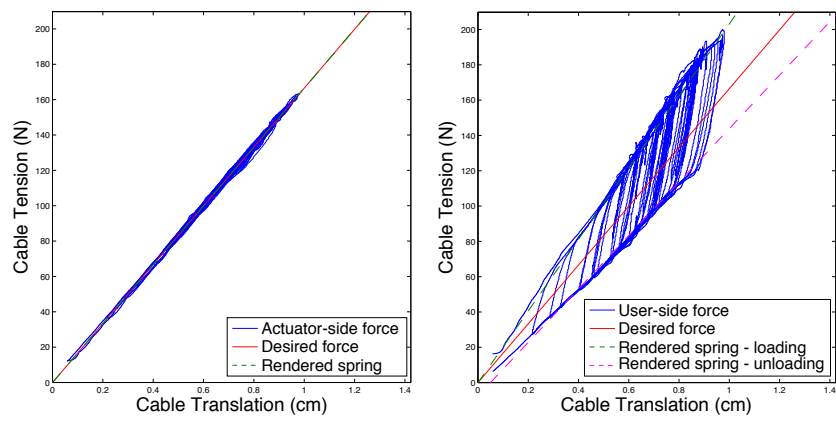

(b)

Fig. 8. Rendered impedance at the user interface for (a) $k_{v}=11.10 \mathrm{~N} / \mathrm{cm}$ and (b) $k_{v}=166.52 \mathrm{~N} / \mathrm{cm}$. Stiffness is plotted as cable tension versus cable translation. The solid line displays the desired stiffness for the test. Left shows motor side measurement, right shows measurement at user interface. Dashed lines shows fitted stiffness, split in two for user side measurements.

mount to ground no longer extends across the actuated degree of freedom, allowing the joint to be flexible, and 2) it is more robust to disturbances introduced by the user - a fact that is much more relevant when considering the soft exosuit application instead of this idealized test bed. However, with this sensor placement we cannot remove nonlinear cable-conduit friction with force feedback control, as is demonstrated in Fig. 8. When integrating this actuation system into a wearable exosuit, it should be known ahead of time whether or not this error is tolerable.

\section{REFERENCES}

[1] A. Schiele, "Ergonomics of exoskeletons: Objective performance metrics," in IEEE 3rd Joint EuroHaptics Conf. and Symp. Haptic Interfaces for Virtual Environment and Teleoperator Systems. IEEE, 2009, pp. 103-108.

[2] L. Dipietro, A. M. M. Sabatini, and P. Dario, "A survey of glovebased systems and their applications," IEEE Trans. Syst. Man Cybern., vol. 38, no. 4, pp. 461-482, Jul. 2008.

[3] D. E. Nathan and M. J. Johnson, "Design of a grasp assistive glove for adl-focused, robotic assisted therapy after stroke," in IEEE Int. Conf. Rehab. Robot., 2007, pp. 943-950.

[4] L. Connelly, Y. Jia, M. L. Toro, M. E. Stoykov, R. V. Kenyon, and D. G. Kamper, "A pneumatic glove and immersive virtual reality environment for hand rehabilitative training after stroke," IEEE Trans. Neural Syst. Rehabil. Eng., vol. 18, no. 5, pp. 551-559, Oct. 2010.

[5] P. Polygerinos, Z. Wang, K. C. Galloway, R. J. Wood, and C. J. Walsh, "Soft robotic glove for combined assistance and at-home rehabilitation," Robotics and Autonomous Syst., vol. 73, pp. 135-143, Nov. 2015.

[6] J. A. Gallego, E. Rocon, J. Ibáñez, J. L. Dideriksen, A. D. Koutsou, R. Paradiso, M. B. Popovic, J. M. Belda-Lois, F. Gianfelici, and D. Farina, "A soft wearable robot for tremor assessment and suppression," in IEEE Int. Conf. on Robot. and Autom., 2011, pp. 2249-2254.
[7] A. T. Asbeck, S. M. De Rossi, K. G. Holt, and C. J. Walsh, "A biologically inspired soft exosuit for walking assistance," Int. J. Robotics Research, vol. 34, no. 6, pp. 744-762, 2015.

[8] L. M. Mooney and H. M. Herr, "Biomechanical walking mechanisms underlying the metabolic reduction caused by an autonomous exoskeleton," J. Neuroeng. Rehab., vol. 13, no. 1, p. 4, 2016.

[9] J. Bae, S. M. M. De Rossi, K. O'Donnell, K. L. Hendron, L. N. Awad, T. R. T. Dos Santos, V. L. De Araujo, Y. Ding, K. G. Holt, and T. D. Ellis, "A soft exosuit for patients with stroke: feasibility study with a mobile off-board actuation unit," in IEEE Int. Conf, on Rehab. Robotics, 2015, pp. 131-138.

[10] A. T. Asbeck, S. M. De Rossi, I. Galiana, Y. Ding, and C. J. Walsh, "Stronger, smarter, softer: Next-generation wearable robots," IEEE Robot. Automat. Mag., vol. 21, no. 4, pp. 22-33, Dec. 2014.

[11] K. Kong, J. Bae, and M. Tomizuka, "A cable-driven human assistive system and its impedance compensation by sensor fusion," in ASME Dynamic Syst. and Control Conf., 2010, pp. 835-842.

[12] G. A. Pratt and M. M. Williamson, "Series elastic actuators," in IEEE Int. Conf. Intelligent Robots and Syst., vol. 1, 1995, pp. 399-406.

[13] J. F. Veneman, R. Ekkelenkamp, R. Kruidhof, F. C. T. Van der Helm, and H. Van der Kooij, "Design of a series elastic-and bowden cablebased actuation system for use as torque-actuator in exoskeleton-type training," in IEEE Int. Conf. Rehab. Robot., 2005, pp. 496-499.

[14] P. Agarwal, J. Fox, Y. Yun, M. K. O’Malley, and A. D. Deshpande, “An index finger exoskeleton with series elastic actuation for rehabilitation: Design, control and performance characterization," Int. J. Robot. Research, vol. 34, no. 14, pp. 1747-1772, Oct. 2015.

[15] L. Cappello, A. Pirrera, P. Weaver, and L. Masia, "A series elastic composite actuator for soft arm exosuits," in IEEE Int. Conf. on Rehab. Robot., 2015, pp. 61-66.

[16] J. E. Colgate, "The control of dynamically interacting systems," $\mathrm{Ph} . \mathrm{D}$. dissertation, Massachusetts Institute of Technology, 1988.

[17] R. C. Browning, J. R. Modica, R. Kram, and A. Goswami, "The effects of adding mass to the legs on the energetics and biomechanics of walking," Medicine and Science in Sports and Exercise, vol. 39, no. 3, p. $515,2007$.

[18] F. A. Panizzolo, I. Galiana, A. T. Asbeck, C. Siviy, K. Schmidt, K. G Holt, and C. J. Walsh, "A biologically-inspired multi-joint soft exosuit that can reduce the energy cost of loaded walking," J. Neuroeng. and Rehabil., vol. 13, no. 1, p. 43, 2016.

[19] J. Rosen, J. C. Perry, N. Manning, S. Burns, and B. Hannaford, "The human arm kinematics and dynamics during daily activities-toward a 7 dof upper limb powered exoskeleton," in IEEE Int. Conf. Advanced Robotics, 2005, pp. 532-539.

[20] D. J. Bennett, J. M. Hollerbach, Y. Xu, and I. W. Hunter, "Timevarying stiffness of human elbow joint during cyclic voluntary movement," Experimental Brain Research, vol. 88, no. 2, pp. 433-442, 1992.

[21] M. M. Williamson, "Series elastic actuators," Ph.D. dissertation, Massachusetts Institute of Technology, 1995.

[22] F. Sergi, M. M. Lee, and M. K. O'Malley, "Design of a series elastic actuator for a compliant parallel wrist rehabilitation robot," in IEEE Int. Conf. Rehab. Robot., 2013, pp. 1-6.

[23] F. Sergi and M. K. O'Malley, "On the stability and accuracy of high stiffness rendering in non-backdrivable actuators through series elasticity," Mechatronics, vol. 26, pp. 64-75, Mar. 2015.

[24] D. W. Robinson, "Design and analysis of series elasticity in closedloop actuator force control," Ph.D. dissertation, Massachusetts Institute of Technology, 2000.

[25] D. P. Losey, A. Erwin, C. G. McDonald, F. Sergi, and M. K. O'Malley, "A time-domain approach to control of series elastic actuators: Adaptive torque and passivity-based impedance control," IEEE Trans. Mechatron., vol. 21, no. 4, pp. 2085-2096, Aug. 2016.

[26] M. Kaneko, T. Yamashita, and K. Tanie, "Basic considerations on transmission characteristics for tendon drive robots," in Int. Conf. Advanced Robot., vol. 1, Jun. 1991, pp. 827-832.

[27] V. Agrawal, W. J. Peine, and B. Yao, "Modeling of a closed loop cableconduit transmission system," in IEEE Int. Conf. Robot. and Autom., 2008, pp. 3407-3412. 